\title{
Photoacoustic computed tomography guided microrobots for targeted navigation in intestines in vivo
}

Li, Lei, Wu, Zhiguang, Yang, Yiran, Hu, Peng, Gao, Wei, et al.

Lei Li, Zhiguang Wu, Yiran Yang, Peng Hu, Wei Gao, Lihong V. Wang, "Photoacoustic computed tomography guided microrobots for targeted navigation in intestines in vivo," Proc. SPIE 11240, Photons Plus Ultrasound: Imaging and Sensing 2020, 112402R (17 February 2020); doi: $10.1117 / 12.2547559$

SPIE. Event: SPIE BiOS, 2020, San Francisco, California, United States 


\title{
Photoacoustic computed tomography guided microrobots for targeted navigation in intestines in vivo
}

\author{
Lei $\mathrm{Li}^{\mathrm{a}}$, Zhiguang $\mathrm{Wu}^{\mathrm{a}}$, Yiran Yang ${ }^{\mathrm{a}}$, Peng $\mathrm{Hu}^{\mathrm{a}}$, Wei Gao ${ }^{\mathrm{a} *}$ and Lihong V. Wang ${ }^{\mathrm{a} *}$ \\ aAndrew and Peggy Cherng Department of Medical Engineering, California Institute of Technology, \\ 1200 E California Blvd., Pasadena, CA 91125
}

${ }^{*}$ Correspondence should be addressed to:

W. G. (weigao@caltech.edu) and L.V.W. (LVW@caltech.edu)

\begin{abstract}
Tremendous progress in synthetic micro/nanomotors has been made for potential biomedical applications. However, existing micro/nanomotor platforms are inefficient for deep tissue imaging and motion control in vivo. Here, we present a photoacoustic computed tomography (PACT) guided investigation of micromotors in intestines in vivo. The micromotors enveloped in microcapsules exhibit efficient propulsion in various biofluids once released. PACT has visualized the migration of micromotor capsules toward the targeted regions in real time in vivo. The integration of the developed microrobotic system and PACT enables deep imaging and precise control of the micromotors in vivo.
\end{abstract}

Keywords: Photoacoustic computed tomography; microrobots; in vivo navigation

\section{INTRODUCTION}

Micro and nanorobots that can be navigated into hard-to-reach tissues have drawn extensive attention for the promise to empower various biomedical applications, such as disease diagnosis, targeted drug delivery, and minimally invasive surgery [1-3]. Chemically powered motors, in particular, show great potential toward in vivo application thanks to their autonomous propulsion and versatile functions in bodily fluids [4-6]. However, imaging and control of micromotors in vivo remain major challenges for practical medical investigations, despite the significantly advanced development of micromotors [7-9]. The ability to directly visualize the dynamics of micromotors with high spatiotemporal resolution in vivo at the whole-body scale is in urgent demand to provide real-time visualization and guidance of micromotors [8]. In addition to high spatiotemporal resolution, the ideal non-invasive micromotor imaging technique should offer deep penetration and molecular contrasts.

To date, optical imaging is widely used for biomedical applications owing to its high spatiotemporal resolution and molecular contrasts.[10-12] However, applying conventional optical imaging to deep tissues is hampered by strong optical scattering, which inhibits high-resolution imaging beyond the optical diffusion limit ( 1-2 mm in depth) [13-15]. Fortunately, photoacoustic (PA) tomography (PAT), detecting photon-induced ultrasound, achieves high-resolution imaging at depths that far exceed the optical diffusion limit [16]. In PAT, the energy of photons absorbed by chromophores inside the tissue is converted to acoustic waves, which are subsequently detected to yield high-resolution tomographic images with optical contrasts. Leveraging the negligible acoustic scattering in soft tissue, PAT has achieved superb spatial resolution at depths, with a depth-to-resolution ratio of 200 [12, 17], at high imaging rates. As a major incarnation of PAT, photoacoustic computed tomography (PACT) has attained high spatiotemporal resolution, deep penetration, and anatomical and molecular contrasts [18-22]. With all these benefits, PACT shows great promise for real-time navigation of micromotors in vivo for broad applications, particularly, drug delivery. Drug delivery through the gastrointestinal (GI) tract serves as a convenient and versatile therapeutic tool, owing to its cost-effectiveness, high patient compliance, lenient constraint for sterility, and ease of production [23]. Although oral administration of various micro/nanoparticle-based drug delivery systems has been demonstrated to both survive the acidic gastric environment and diffuse into the intestines, drug absorption is still inefficient due to the limited intestinal retention time [24]. A large number of passive diffusion-based targeting strategies have been explored to improve the delivery efficiency, but they suffer from low precision, size restraint and specific surface chemistry [25]. With precise control, microrobotic drug delivery systems can potentially achieve targeted delivery with long retention times and sustainable release profiles, which are in pressing need [26]. Due to the lack of imaging-guided control, there is no report yet for precisely targeted

Photons Plus Ultrasound: Imaging and Sensing 2020, edited by Alexander A. Oraevsky, Lihong V. Wang, Proc. of SPIE Vol. 11240, 112402R - @ 2020 SPIE · CCC code: 1605-7422/20/\$21 · doi: 10.1117/12.2547559 
delivery using micromotors in vivo [8]. Additionally, biocompatibility is required, and an ideal microrobotic system is expected to be cleared safely by the body after completion of the tasks [2].

In this paper, we describe a PACT-guided microrobotic system (PAMR), which has accomplished controlled propulsion and prolonged cargo retention in vivo. Owing to the high spatiotemporal resolution, non-invasiveness, molecular contrast, and deep penetration, PACT provides an attractive tool to locate and navigate the micromotors in vivo [27, 28]. Ingestible Mg-based micromotors are encapsulated in enteric protective capsules to prevent reactions in gastric acid and allow direct visualization by PACT. PACT monitors the migration of micromotor capsules (MCs) in intestines in real time; continuous-wave (CW) near-infrared (NIR) light irradiation induces phase transition of microcapsules and triggers propulsion of the micromotors; the autonomous and efficient propulsion of the micromotors enhances the retention in targeted areas of the GI tract. We believe that the proposed integrated microrobotic system will substantially advance gastrointestinal therapies.

\section{METHODS}

The fabrication of MCs mainly consists of two steps: the fabrication of Mg-based micromotors (Fig. 1a) and the formation of MCs (Fig. 1b). In the first step (Fig. 1a), Mg microparticles with a diameter of $\sim 20 \mu \mathrm{m}$ were dispersed onto glass slides, followed by the deposition of a gold layer, which facilitates the autonomous chemical propulsion in GI fluids and enhances PA contrast of the micromotors. An alginate hydrogel layer was coated onto the micromotors by dropping aqueous solution containing alginate and drugs (e.g., doxorubicin) on the slides. A parylene layer, acting as a shell scaffold that ensures the stability during propulsion, was then deposited onto the micromotors. A small opening ( $\sim 2$ $\mu \mathrm{m}$ in diameter), attributed to the surface contact of the Mg microparticles with the glass slides during various layer coating steps, acts as a catalytic interface for gas propulsion in the intestinal environment. Next, the micromotors were encapsulated into the enteric gelatin capsules by the emulsion method (Fig. 1b).

(a)

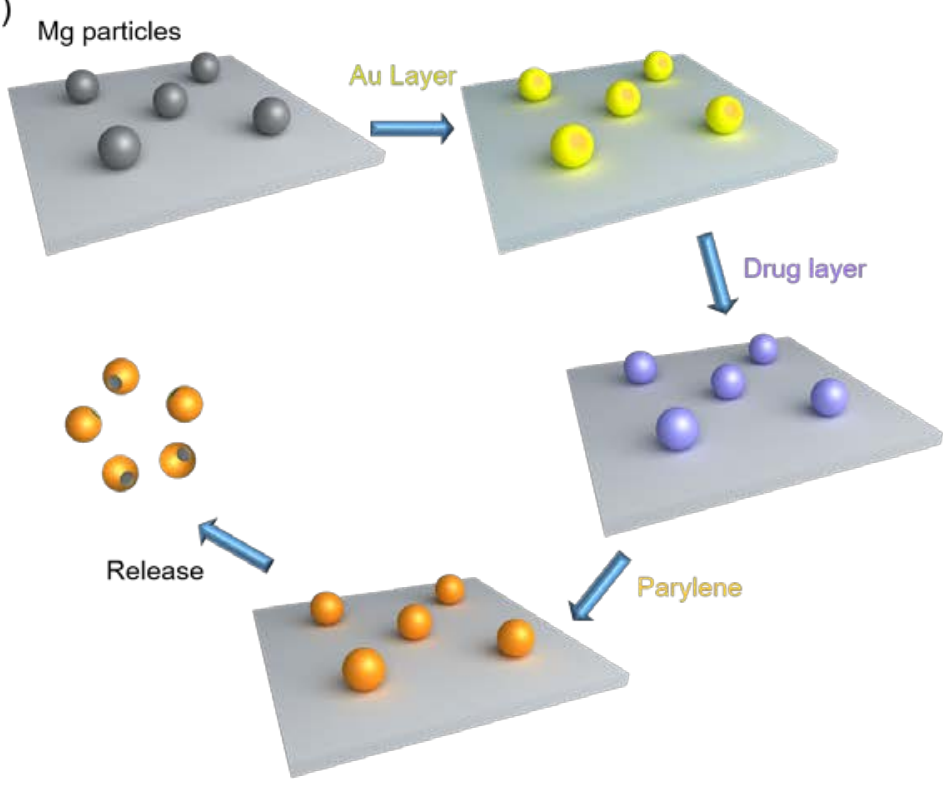

(b)

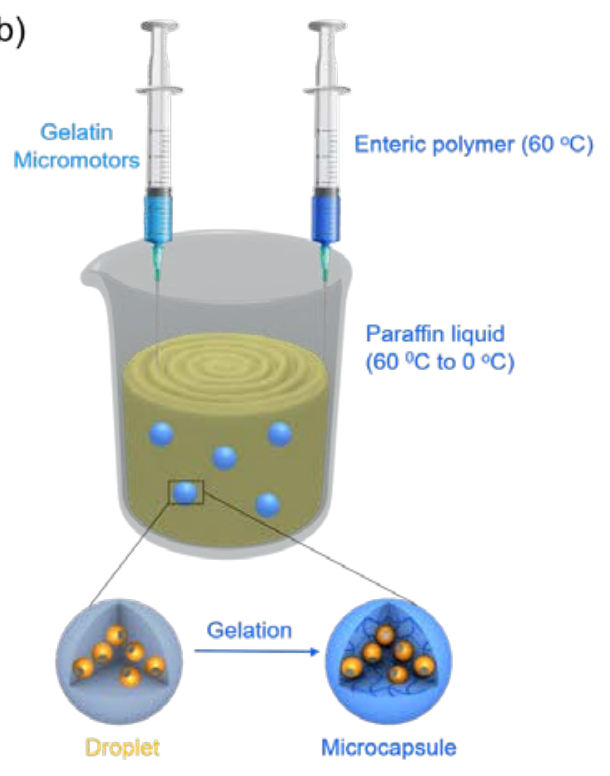

Figure 1. (a) The fabrication flow of the ingestible micromotors. (b) The preparation of the MCs.

The green fluorescence from the fluorescein isothiocyanate-labeled bovine serum albumin (FITC-albumin) and red fluorescence from doxorubicin (DOX) were observed from the micromotors (Fig. 2, top row) and the MCs (Fig. 2, bottom row), confirming a successful drug loading. 

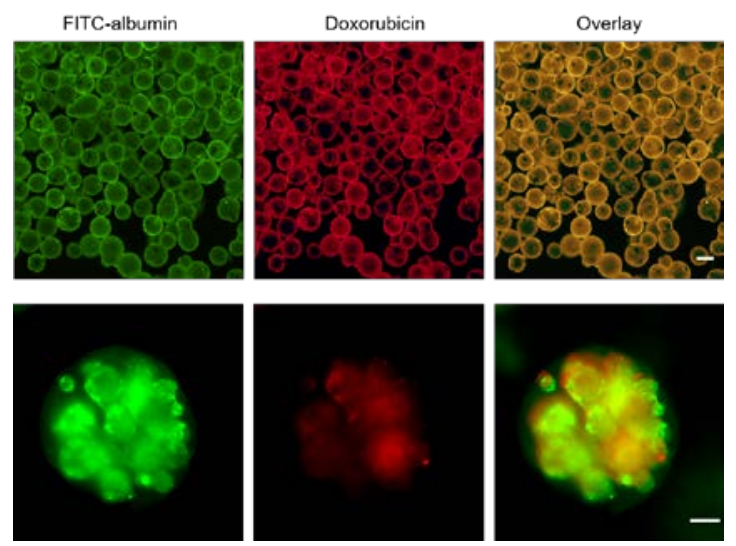

Figure 2. Fluorescence images of the micromotors (top row) and the MCs (bottom row), confirming the successful drug loading. Scale bar, $20 \mu \mathrm{m}$.

For deep tissue imaging in vivo, it is crucial that the MCs should have higher optical absorption than the blood background. To evaluate the PA imaging performance of the MCs, the PA amplitudes of the MCs, whole blood, and bare $\mathrm{Mg}$ particles were measured. NIR light experiences the least attenuation in mammalian tissues, permitting the deepest optical penetration. As shown in Fig. 3a, the MCs exhibit strong PA contrast in the NIR wavelength region, ranging from 720 to $890 \mathrm{~nm}$. At the wavelength of $750 \mathrm{~nm}$, the MCs display the highest PA amplitude of 15.3 (Fig. 3a). The bare Mg particles display a similar PA spectrum, with a lower PA peak with an amplitude of 10.0 at $750 \mathrm{~nm}$. The difference due to the Au layer is expected to significantly improve the imaging sensitivity in the NIR wavelength region (Fig. 3a) [29-31]. In addition, the approximate 3-fold increase in PA amplitudes of the MCs compared to that of the whole blood provides sufficient contrast for PACT to detect the MCs in vivo using 750-nm illumination. To evaluate the stability of the MCs under pulsed NIR PA excitation, we measured the PA signal fluctuation of the MCs during PA imaging (Fig. 3b). We also studied the maximum detectable depth of MCs using PACT (Fig. 3c). The micromotors showed dramatically decreased fluorescence intensity when covered by thin tissues (0.7-2.4 mm in thickness) and became undetectable quickly (Fig. 3c, inset). By contrast, PACT can image the micromotors inside tissue as deep as $\sim 7$ $\mathrm{cm}$ (Fig. 3c), which reveals that the key advantage of PACT lies in the high spatial resolution and high molecular contrast for deep imaging in tissues [18].
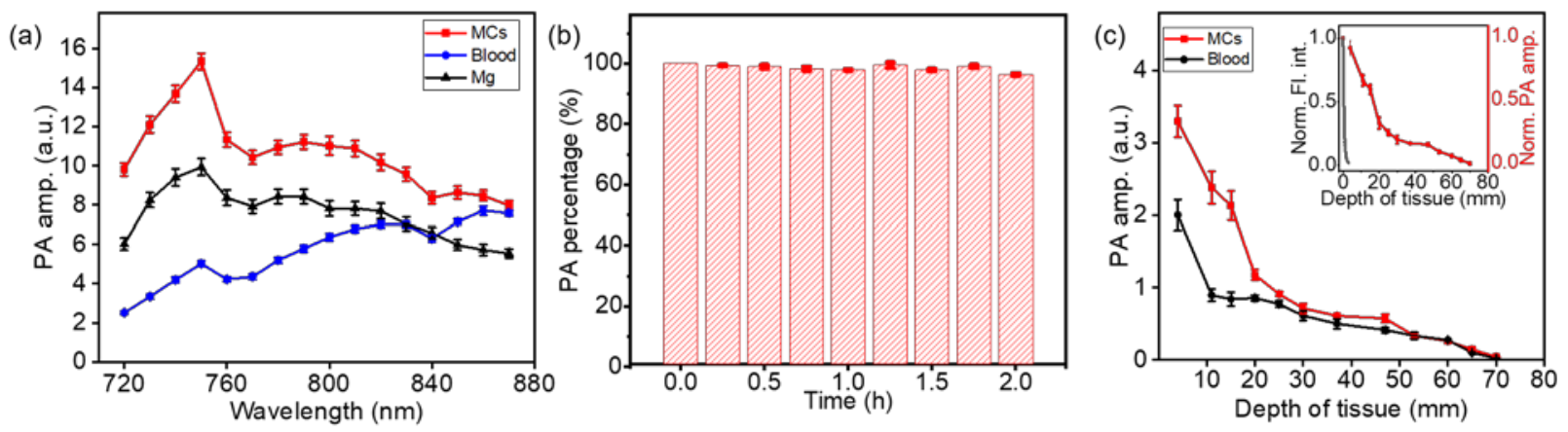

Figure 3. Characterization of the MCs. (a) PACT spectra of MCs (red line), blood (blue line), and Mg particles (black line). (b) Long-term stability of the PA signals of the MCs under the NIR illumination used in the PACT. Error bars represent the SDs from 5 independent measurements. (c) Dependence of PA amplitude of the MCs (red line) and blood (black line) on the depth of tissue and the normalized PA amplitude and fluorescence intensity of the MCs under tissues (inset). Norm., normalized; amp., amplitude; Fl., fluorescence; int., intensity. Error bars represent the SDs from five independent measurements.

\section{RESULTS}

The movement of a swarm of MCs was monitored in vivo by PACT. The MCs were dispersed in pure water and then orally administered into 5-6-week old nude mice. The mice were subsequently anesthetized, and the lower abdominal 
cavity was aligned with the imaging plane of the ultrasonic transducer array for longitudinal imaging. PACT images were captured at a frame rate of $2 \mathrm{~Hz}$ for $\sim 8$ hours. As shown in Fig. 4a, where the blood vessels and background tissues are shown in gray and MCs in intestines are highlighted in color. During the imaging period of the first 6 hours, the MCs migrated for $\sim 1.2 \mathrm{~cm}$, roughly $15 \%$ of the length of the entire small intestine. After 5 hours, the PA signals of some MCs faded away as they moved downstream in intestines that were outside of the imaging plane. The moving speed of the

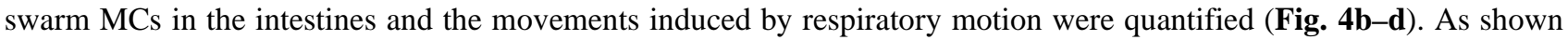
in Fig. $\mathbf{4 b} \mathbf{b}-\mathbf{d}$, the abrupt motion caused by respiration is much faster than the actual migration of the MCs. Despite the respiration-induced movement, PACT can distinguish the signals from the slowly migrating MCs in the intestines. These results indicate that PACT can precisely monitor and track the locations of the MCs in deep tissues in vivo.


(b)

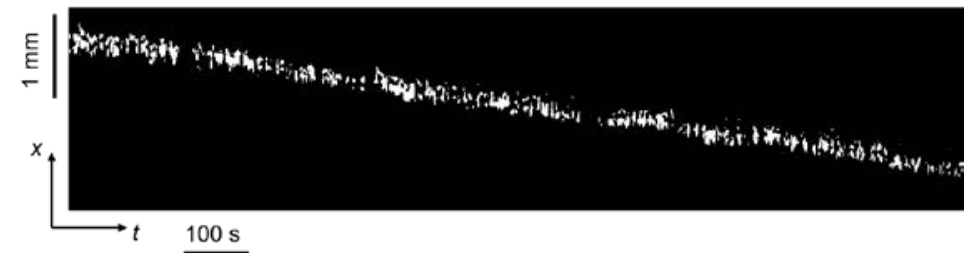

(c)

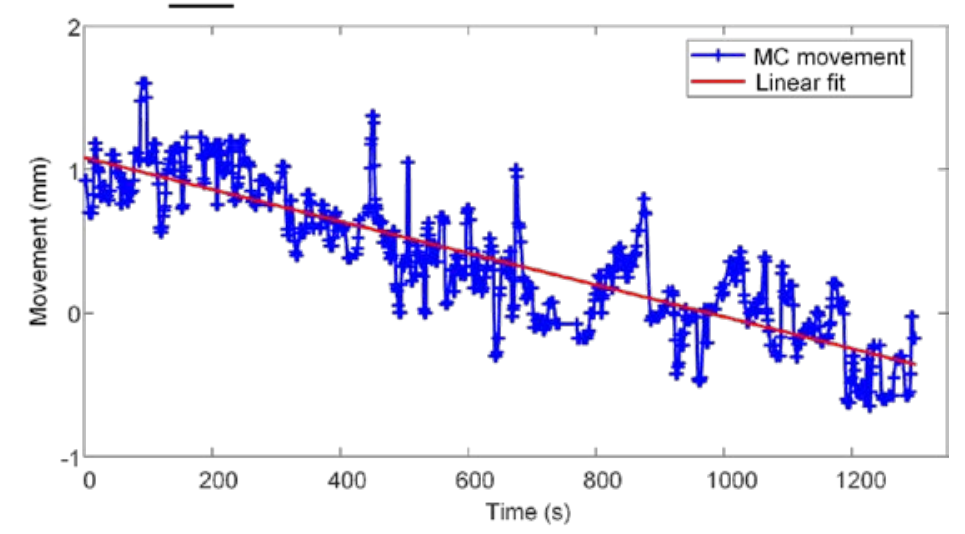

Figure 4. PACT evaluation of the MCs dynamics in vivo. (a) A representative PACT image of the MCs in intestines in vivo. The MCs migrating in the intestine are shown in color; the mouse tissues are shown in gray. Scale bar, $2 \mathrm{~mm}$. (b) The thresholded $x$ - $t$ image showing the segmented MCs at elapsed time. (c) The movement displacement caused by the migration of the MCs in intestines. (d) Comparison of the speeds of the MC migration and the respiration-induced movement. Error bars represent the SDs from three independent measurements.

Of particular biomedical significance is the retention of cargo carriers in the targeted region in intestines. While most of the previous studies focused on improving the interactions between particles and the mucoadhesives by engineering surface coatings on the passive particles, the biofluid-driven propulsion of the active micromotors can dramatically prolong their retention in intestine walls. When the MCs approach the targeted areas in intestines, we can trigger the collapse of the capsules and activate the propulsion of micromotors on demand (Fig. 5a). Once the MCs approach the targeted location, they are irradiated with CW NIR light to trigger a responsive release of the micromotors. The PA signals from the MCs in the intestines were prolonged upon the CW NIR irradiation, suggesting the release of the micromotors (Fig. 5b). Besides the active propulsion, the enhanced retention in vivo may also be attributed to the elevated $\mathrm{pH}$ and $\mathrm{Mg}^{2+}$ concentration in the surrounding environment caused by Mg-water reactions. It has been recently reported that high $\mathrm{pH}(\sim 8.2-12.0)$ could trigger a phase transition of the mucus and facilitate tissue penetration of the micro/nanoparticles [32]. To investigate the influence of the micromotors on the $\mathrm{pH}$ of the surrounding environment, the 
micromotors were dispersed in water with phenolphthalein as a $\mathrm{pH}$ indicator. The microscopic image in Fig. 5c shows red/orange color in the vicinity of a micromotor, indicating an increased $\mathrm{pH}$ of the surrounding medium.
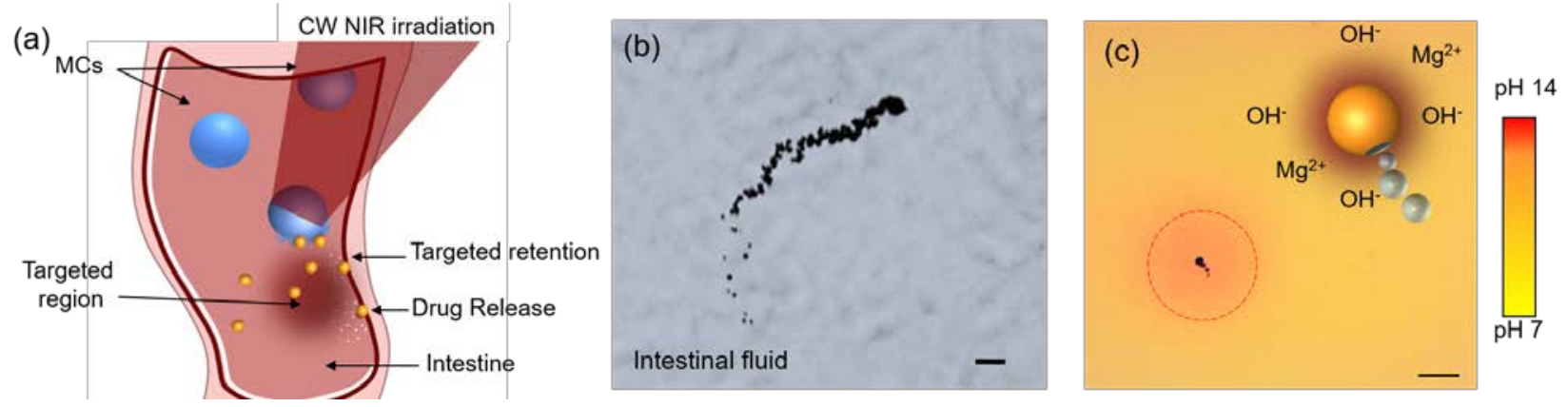

Figure 5. Evaluation of the MCs for targeted retention and delivery. (a) Schematic of the use of the MCs for targeted delivery in intestines. (b) Microscopic images showing the propulsion of the micromotor in intestinal fluid after CW NIR triggered release. Scale bar, $20 \mu \mathrm{m}$. (c) Microscopic image displaying the change of $\mathrm{pH}$ of the surrounding environment upon the micromotors in PBS.

\section{DISCUSSION}

Two key challenges should be addressed for applying synthetic micromotors to practical biomedical applications: 1) advanced imaging techniques to locate micromotors in deep tissue at high spatiotemporal resolution with high contrast; 2) precise on-demand control of micromotors in vivo. With high molecular sensitivity at depths, PACT allows real-time monitoring of micromotors in intestines at high spatial resolution for subsequent control. Here, micromotors with partial coating of functional multilayers are designed as both the imaging contrast agents and the controllable drug carriers. An Au layer is employed to significantly increase the optical absorption for PA imaging and the reaction rate for efficient propulsion simultaneously. A gelatin hydrogel layer is used to enlarge the loading capacity of different functional components, such as therapeutic drugs and imaging agents. A parylene layer is applied to maintain the geometry of the micromotors during propulsion. Our current platform, integrating real-time imaging and control of micromotors in intestines in vivo, leads to the next generation of intelligent microrobotic systems, and provides opportunities for precise microsurgery and targeted drug delivery.

Although the current platform has been demonstrated in small animals, human clinical translations may require tens of centimeters of tissue penetration. PACT can provide up to $7-\mathrm{cm}$ tissue penetration, which is limited by photon dissipation [21, 33]. By employing a more penetrating excitation source-microwave and acoustic detection, thermoacoustic tomography (TAT) promises tissue penetration for clinical translations [34]. Moreover, incorporation of a gold layer in the micromotor design provides an excellent microwave absorption contrast for TAT owing to the high electrical conductivity, and thus greatly enhances the deep tissue imaging capability of the microrobots for clinical applications. Focused ultrasound heating can increase the depths of thermally triggered microrobot release to the wholebody level of humans. Currently, the passive diffusion-based delivery suffers from complex designs, particle size constraints, low precision, and poor specificity. Our platform allows micromotors to reach any targeted regions in intestines with high precision. It can be tailored to particles of any sizes and can be applied to any biological media without additional design efforts. Our platform can also be easily modified to carry various cargos, including therapeutic agents and diagnostic sensors, with real-time feedback during delivery. Biocompatibility and biodegradability of the micromotors are essential for practical biomedical applications. The components of our micromotors, widely used as therapeutic agents and in implantable devices, are studied to be safe for in vivo applications [35]. The micromotors have been eventually cleared by the digestive system via excrement, without any adverse effects.

In summary, we report an ingestible microrobotic platform with high optical absorption for imaging-assisted control in intestines. The encapsulated micromotors survive the erosion of the stomach fluid and permit propulsion in intestines. PACT non-invasively monitors the migration of the micromotors and visualizes their arrival at targeted areas in vivo. CW NIR irradiation toward targeted areas induces a phase transition of the capsules and triggers the propulsion of the micromotors. The mechanical propulsion provides a driving force for the micromotors to bind to the intestine walls, resulting in an extended retention. The proposed platform lays a foundation for targeted delivery in tissues and opens a new horizon for precision medicine. 
This work was sponsored by the Startup funds from California Institute of Technology (to W.G.) and the NIH grants EB016986 (NIH Director's Pioneer Award), CA186567 (NIH Director's Transformative Research Award), NS090579, and NS099717 (all to L.V.W.). We gratefully acknowledge critical support and infrastructure provided for this work by the Kavli Nanoscience Institute at Caltech.

\section{REFERENCES}

[1] P. Fischer, B. J. Nelson, and G.-Z. Yang, "New materials for next-generation robots,” 3(18), eaau0448 (2018).

[2] X. Yan, Q. Zhou, M. Vincent et al., "Multifunctional biohybrid magnetite microrobots for imaging-guided therapy,” 2(12), eaaq1155 (2017).

[3] Z. Wu, L. Li, Y. Yang et al., "A microrobotic system guided by photoacoustic computed tomography for targeted navigation in intestines in vivo," Science Robotics, 4(32), eaax0613 (2019).

[4] S. Sánchez, L. Soler, and J. Katuri, “Chemically Powered Micro- and Nanomotors,” 54(5), 1414-1444 (2015).

[5] J. Wang, and W. Gao, "Nano/Microscale Motors: Biomedical Opportunities and Challenges," ACS Nano, 6(7), 5745-5751 (2012).

[6] W. Gao, R. Dong, S. Thamphiwatana et al., "Artificial Micromotors in the Mouse's Stomach: A Step toward in Vivo Use of Synthetic Motors,” ACS Nano, 9(1), 117-123 (2015).

[7] T. Li, X. Chang, Z. Wu et al., "Autonomous Collision-Free Navigation of Microvehicles in Complex and Dynamically Changing Environments,” ACS Nano, 11(9), 9268-9275 (2017).

[8] M. S. Medina-Sánchez, Oliver G "Medical microbots need better imaging and control,” Nature, 545(7655), 406-408 (2017).

[9] D. Vilela, U. Cossío, J. Parmar et al., "Medical Imaging for the Tracking of Micromotors," ACS Nano, 12(2), 1220-1227 (2018).

[10] J. Yang, L. Li, A. A. Shemetov et al., "Focusing light inside live tissue using reversibly switchable bacterial phytochrome as a genetically encoded photochromic guide star,” Science Advances, 5(12), eaay1211 (2019).

[11] Y. Li, L. Li, L. Zhu et al., "Snapshot photoacoustic topography through an ergodic relay for high-throughput imaging of optical absorption,” Nature Photonics, 1-7 (2020).

[12] L. Li, A. A. Shemetov, M. Baloban et al., "Small near-infrared photochromic protein for photoacoustic multicontrast imaging and detection of protein interactions in vivo,” Nature communications, 9(1), 2734 (2018).

[13] P. Zhang, L. Li, L. Lin et al., "In vivo superresolution photoacoustic computed tomography by localization of single dyed droplets,” Light: Science \& Applications, 8(1), 36 (2019).

[14] J. Shi, T. T. Wong, Y. He et al., "High-resolution high-contrast mid-infrared imaging of fresh biological samples with ultraviolet-localized photoacoustic microscopy (Conference Presentation)." 10878, 1087825.

[15] T. P. Matthews, J. Poudel, L. Li et al., "Parameterized Joint Reconstruction of the Initial Pressure and Sound Speed Distributions for Photoacoustic Computed Tomography,” SIAM Journal on Imaging Sciences, 11(2), 1560-1588 (2018).

[16] P. Zhang, L. Li, L. Lin et al., "High - resolution deep functional imaging of the whole mouse brain by photoacoustic computed tomography in vivo,” Journal of biophotonics, 11(1), e201700024 (2018).

[17] L. H. V. Wang, and S. Hu, "Photoacoustic tomography: in vivo imaging from organelles to organs," Science, 335(6075), 1458-1462 (2012).

[18] L. Li, L. Zhu, C. Ma et al., "Single-impulse panoramic photoacoustic computed tomography of small-animal whole-body dynamics at high spatiotemporal resolution,” Nature Biomedical Engineering, 1, 0071 (2017).

[19] J. Yao, A. A. Kaberniuk, L. Li et al., "Multiscale photoacoustic tomography using reversibly switchable bacterial phytochrome as a near-infrared photochromic probe," Nature Methods, 13, 67 (2015).

[20] Y. Qu, L. Li, Y. Shen et al., "Dichroism-sensitive photoacoustic computed tomography,” Optica, 5(4), 495-501 (2018).

[21] L. Li, J. Xia, G. Li et al., "Label-free photoacoustic tomography of whole mouse brain structures ex vivo," Neurophotonics, 3(3), 035001 (2016).

[22] L. Zhu, L. Li, L. Gao et al., "Multiview optical resolution photoacoustic microscopy," Optica, 1(4), $217-222$ (2014).

[23] M. Koziolek, M. Grimm, F. Schneider et al., "Navigating the human gastrointestinal tract for oral drug delivery: Uncharted waters and new frontiers,” Advanced Drug Delivery Reviews, 101, 75-88 (2016). 
[24] K. S. Soppimath, A. R. Kulkarni, W. E. Rudzinski et al., "MICROSPHERES AS FLOATING DRUGDELIVERY SYSTEMS TO INCREASE GASTRIC RETENTION OF DRUGS,” Drug Metabolism Reviews, 33(2), 149-160 (2001).

[25] D. Rosenblum, N. Joshi, W. Tao et al., "Progress and challenges towards targeted delivery of cancer therapeutics,” Nature Communications, 9(1), 1410 (2018).

[26] G.-Z. Yang, J. Bellingham, P. E. Dupont et al., "The grand challenges of <em>Science Robotics</em>,” 3(14), eaar7650 (2018).

[27] C. Yeh, L. Li, L. Zhu et al., "Dry coupling for whole-body small-animal photoacoustic computed tomography,” Journal of biomedical optics, 22(4), 041017 (2017).

[28] L. Li, L. Zhu, Y. Shen et al., "Multiview Hilbert transformation in full-ring transducer array-based photoacoustic computed tomography,” Journal of biomedical optics, 22(7), 076017 (2017).

[29] T. Ji, V. G. Lirtsman, Y. Avny et al., "Preparation, Characterization, and Application of Au-Shell/Polystyrene Beads and Au-Shell/Magnetic Beads,” 13(16), 1253-1256 (2001).

[30] M. Zhou, L. Li, J. Yao et al., [Nanoparticles for Photoacoustic Imaging of Vasculature] Springer, Cham, (2017).

[31] L. Li, J. Yao, and L. V. Wang, [Photoacoustic tomography enhanced by nanoparticles] John Wiley \& Sons, Inc., (2016).

[32] J. Leal, H. D. C. Smyth, and D. Ghosh, "Physicochemical properties of mucus and their impact on transmucosal drug delivery,” International Journal of Pharmaceutics, 532(1), 555-572 (2017).

[33] B. Rao, R. Zhang, L. Li et al., "Photoacoustic imaging of voltage responses beyond the optical diffusion limit," Scientific reports, 7(1), 2560 (2017).

[34] Y. Xu, and L. H. V. Wang, "Rhesus monkey brain imaging through intact skull with thermoacoustic tomography,” Ieee Transactions on Ultrasonics Ferroelectrics and Frequency Control, 53(3), 542-548 (2006).

[35] N. Sezer, Z. Evis, S. M. Kayhan et al., "Review of magnesium-based biomaterials and their applications," Journal of Magnesium and Alloys, 6(1), 23-43 (2018). 